\title{
Can 3D surgical planning and patient specific instrumentation reduce hip implant inventory? A prospective study
}

\author{
Anna Di Laura ${ }^{1,2^{*}}$, Johann Henckel ${ }^{1}$, Harry Hothi ${ }^{1,2}$ and Alister Hart ${ }^{1,2}$
}

\begin{abstract}
Background: Modern designs of joint replacements require a large inventory of components to be available during surgery. Pre-operative CT imaging aids 3D surgical planning and implant sizing, which should reduce the inventory size and enhance clinical outcome. We aimed to better understand the impact of the use of 3D surgical planning and Patient Specific Instrumentation (PSI) on hip implant inventory.
\end{abstract}

Methods: An initial feasibility study of 25 consecutive cases was undertaken to assess the discrepancy between the planned component sizes and those implanted to determine whether it was possible to reduce the inventory for future cases. Following this, we performed a pilot study to investigate the effect of an optimized inventory stock on the surgical outcome: we compared a group of 20 consecutive cases (experimental) with the 25 cases in the feasibility study (control). We assessed: (1) accuracy of the 3D planning system in predicting size (\%); (2) inventory size changes (\%); (3) intra and post-operative complications.

Results: The feasibility study showed variability within 1 size range, enabling us to safely optimize inventory stock for the pilot study. (1) 3D surgical planning correctly predicted sizes in $93 \%$ of the femoral and $89 \%$ of the acetabular cup components; (2) there was a 61\% reduction in the implant inventory size; (3) we recorded good surgical outcomes with no difference between the 2 groups, and all patients had appropriately sized implants.

Conclusions: 3D planning is accurate in up to $95 \%$ of the cases. CT-based planning can reduce inventory size in the hospital setting potentially leading to a reduction in costs.

Keywords: Total hip Arthroplasty, Preoperative planning, Three-dimensional computerised planning, Implant cost, Implant inventory

\section{Background}

In the United States (US), Total Hip Arthroplasty (THA) has been targeted for cost containment by Centers for Medicare and Medicaid Services (CMS) because of its high cost per procedure and its increasing prevalence [1-4]. The implant is the most expensive supply item for joint replacement $[1,5]$.

\footnotetext{
* Correspondence: anna.laura.14@ucl.ac.uk

${ }^{1}$ The Royal National Orthopaedic Hospital, Brockley Hill, Stanmore, London HA7 4LP, UK

${ }^{2}$ Institute of Orthopaedics and Musculoskeletal Science, University College London, London, UK
}

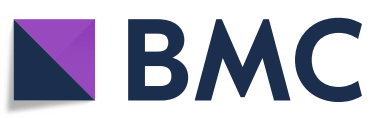

(C) The Author(s). 2020 Open Access This article is licensed under a Creative Commons Attribution 4.0 International License, which permits use, sharing, adaptation, distribution and reproduction in any medium or format, as long as you give appropriate credit to the original author(s) and the source, provide a link to the Creative Commons licence, and indicate if changes were made. The images or other third party material in this article are included in the article's Creative Commons licence, unless indicated otherwise in a credit line to the material. If material is not included in the article's Creative Commons licence and your intended use is not permitted by statutory regulation or exceeds the permitted use, you will need to obtain permission directly from the copyright holder. To view a copy of this licence, visit http://creativecommons.org/licenses/by/4.0/. The Creative Commons Public Domain Dedication waiver (http://creativecommons.org/publicdomain/zero/1.0/) applies to the data made available in this article, unless otherwise stated in a credit line to the data.
Preoperative planning is an essential step in the preparation for elective surgery [6-8]. The technical goals of preoperative planning of the hip joint include restoration of femoral offsets and limb length $[9,10]$ as well as the restoration of the centre of rotation, all of which are dependent on implant size.

Starting from Computed Tomography (CT) scans, Three-Dimensional (3D) models of the patient anatomy are created in a virtual 3D environment. These models are then used to plan the operation, determining implant size, type and positioning in relation to a chosen standard 
frame of reference; the surgeon acquires valuable information regarding patient anatomy before surgery. The digital plan can be transferred to patient care by way of 3D printed personalised instruments and surgical guides, socalled Patient-Specific Instruments (PSI) [11, 12]. 3D printed, sterilised and used intraoperatively, the physical models aid the surgeon achieving optimal cup and stem sizing and positioning [13, 14].

Despite the evidence that CT - based planning (or 3D or virtual planning) is more accurate than conventional 2D radiograph templating in predicting implant size [15-18], it has not been widely adopted. Most 2D planning platforms are integrated into hospital Picture Archiving and Communication System (PACS) systems and are broadly available.

We aimed to better understand the impact of using 3D surgical planning and PSI on inventory size and surgical outcome of THA. Our primary objective was to assess the accuracy of a 3D-CT planning system. Our secondary objective was to evaluate if implant inventory sizes can be reduced with the use of the planning software.

\section{Methods}

\section{Study design}

We designed a prospective study involving a total of 45 consecutive patients undergoing primary cementless THA due to osteoarthritis (OA).

An initial feasibility study (with a group of 25 consecutive cases) was undertaken to assess the discrepancy between the planned component sizes and those implanted, to determine if it was possible to reduce the inventory size for future cases.

Following the feasibility study, we performed a pilot study to investigate the effect of an optimized inventory stock on surgical outcome: we compared a group of 20 consecutive cases (experimental group) with the 25 cases in the feasibility study (control group). The outcome measures were: 1) accuracy of the 3D planning system and PSI in predicting size (\%); 2) inventory size changes (\%) between the two groups; and 3) surgical outcome.

Data collection for the study was performed at our Institution between June 2017 and April 2018 and included patients who underwent primary THA. The surgery was performed through a posterior approach by a single consultant orthopedic surgeon who specializes in hip arthroplasty and has done more than 1000 primary and revision hip arthroplasties.

Prior to surgery, all patients underwent 3D planning with the CT-based software MyHip (Medacta, Castel San Pietro, Switzerland) to determine the size and orientation of the prosthesis most appropriate to restore native hip biomechanics. The MyHip planning system is specifically designed to assist the surgeon with implant selection and positioning and includes the intraoperative use of 3D printed patient-specific guides which can reproduce the surgical plan. 3D printed plastic models of the patient's acetabulum, proximal femur and relative guides, manufactured using 3D printing, are sterilised for intraoperative use. Our institutional review board approved the study (SE16.020).

\section{The patients}

We recruited a consecutive series of 45 patients undergoing primary THA for osteoarthritis. In the initial group selected for the feasibility part of the study there were 14 males and 11 females (mean age 64.36 years, range 39-81); in the experimental group of the pilot study there were 9 males and 11 females (mean age 71.65 years, range 54-79). Table 1.

According to the literature, the accuracy for size component prediction with CT planning is around 90\%, achieved with a minimum of 25 patients (alpha level 0.05 , power of $80 \%)[16,17]$. The number of patients in this study was chosen to provide a reasonable estimate of accuracy as well as reproducibility prior to starting a prospective study $[19,20]$.

\section{CT scanning protocol}

All patients underwent pre-operative CT-scanning of the hip region and the knee joint according to a standard protocol. The scanning protocol is specifically designed to minimize radiation dose, while ensuring a good spatial accuracy. Image acquisition consisted of two short spiral axial scans: one including the whole pelvis (starting at least $2 \mathrm{~cm}$ above the iliac crests and continue to at least $10 \mathrm{~cm}$ below the lesser trochanter) and the proximal femur; the second including the distal part of the femur in the affected side at least $2 \mathrm{~cm}$ above the posterior condyles and extend down to the distal condyles towards the tibia [21].

\section{D surgical planning}

The CT DICOM (Digital Imaging and Communications in Medicine) data was transferred to the MyHip 3D reconstruction planning software to create a patient specific 3D model of the pelvis and femurs of the subject. The engineers at Medacta planned the position and size of the prosthesis according to the surgeon's preferences before he validated the final plan for each case.

MyHip software allows the operator to select a series of bony landmarks on the femur and pelvis in order to define preoperative parameters.

Nine reference points are taken on the femoral side (diaphysis section center, piriformis fossa, anterior tubercle, lesser trochanter, intertrochanteric crest, femoral neck section, center of the femoral head, medial and lateral posterior condyles). Three references are taken on the acetabular side (the acetabular cavity by fitting a sphere, the tear drop and the acetabular north). The relevant axes (femoral anatomical axis, femoral neck axis) and planes (femoral 
Table 1 Patient demographics

\begin{tabular}{llll}
\hline & $\begin{array}{l}\text { Feasibility study group } \\
(\boldsymbol{n}=25)\end{array}$ & Pilot study group $(\boldsymbol{n}=20)$ & $\boldsymbol{p}$-value $($ significant if $<0.05)$ \\
\hline Gender (male: female) & $14: 11$ & $9: 11$ & $p=0.55$ \\
Age at surgery (years) & $64.36( \pm 11.80)$ & $71.65( \pm 7.081)$ & $p=0.01$ \\
Indication (\%) & OA $(100 \%)$ & OA $(100 \%)$ & NA \\
\hline
\end{tabular}

Data presented as ratios, percentages or means $( \pm S D)$

$O A$ osteoarthritis; NA not applicable

neck plane, femoral anteversion plane, anterior pelvic plane and neck cut plane) are defined by the software after anatomical landmarks acquisition; Fig. 1.

Once the operator has selected all landmarks, he proceeds with adjusting the cut height. During the planning of the stem position and of the femoral head resection, all parameters related to the femoral resection in terms of cut height, cut angle and cut anteversion can be modified according to the surgeons' planning philosophy. The stem is positioned in terms of AP (anterior/ posterior) and ML (medial/lateral) offset from the default position, Fig. $2 \mathrm{a}$.
The stem size is chosen to achieve a good fit in the intramedullary canal and head size is selected to adjust the femoral offset. Stem version (Fig. 2b), leg length adjustment, cup positioning can all be adjusted to achieve optimal alignment between the two legs (using the lesser trochanter as a reference). The surgeon defines the acetabular reaming depth and the acetabular angles (anteversion and inclination). Different spatial views are available, the operator can change the size of component among those available, while adjusting the level of fit-and-fill, Fig. 2.

The result of the previous steps can be evaluated in the so-called global view and the leg length view. While

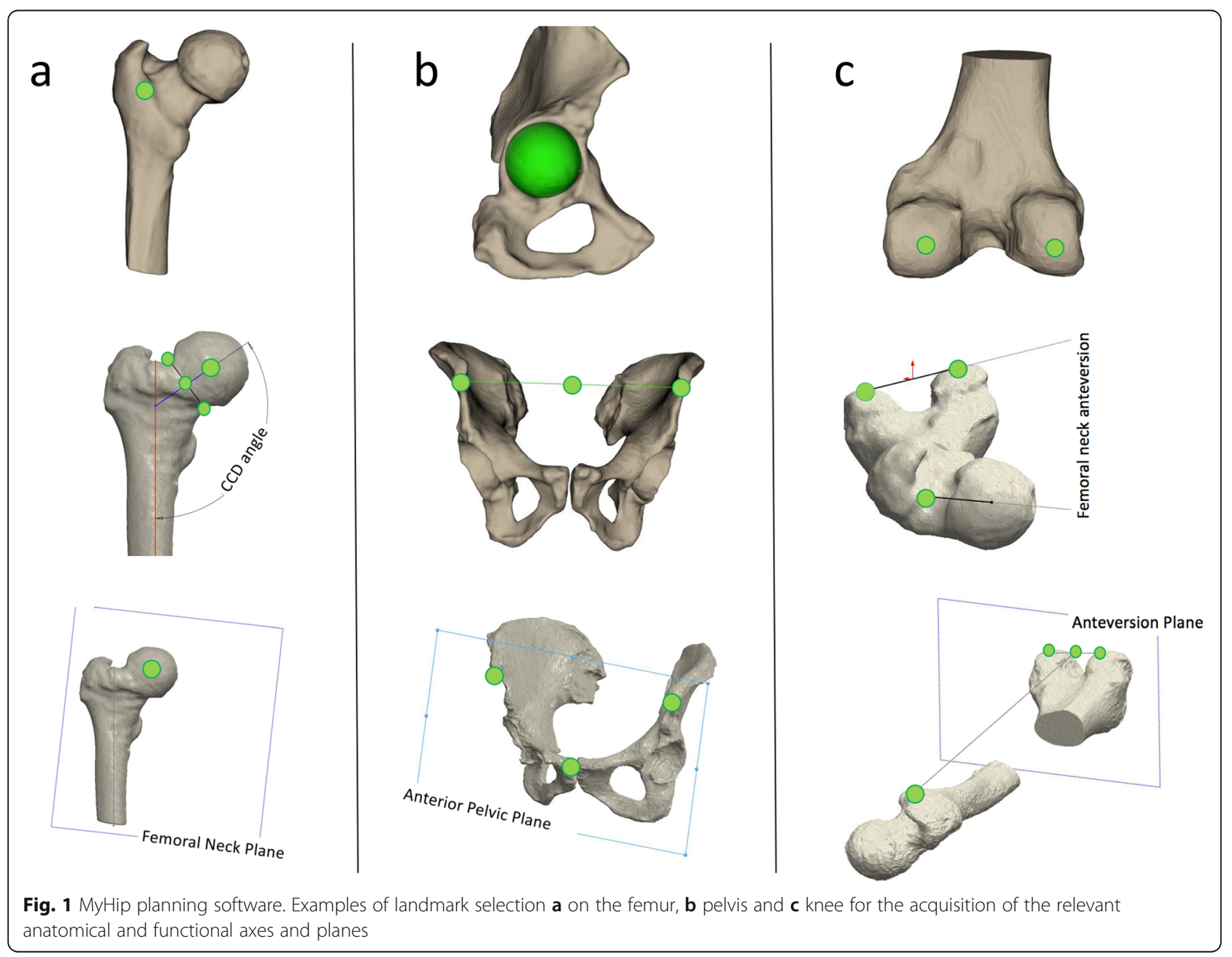




\section{a}

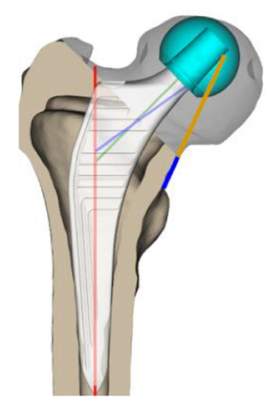

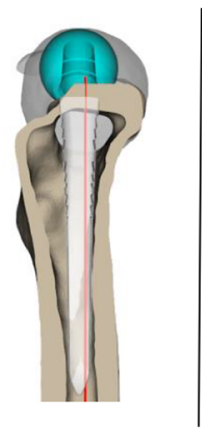

b

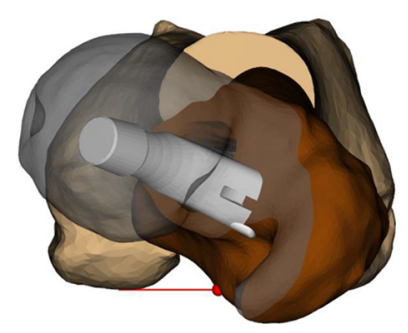

C

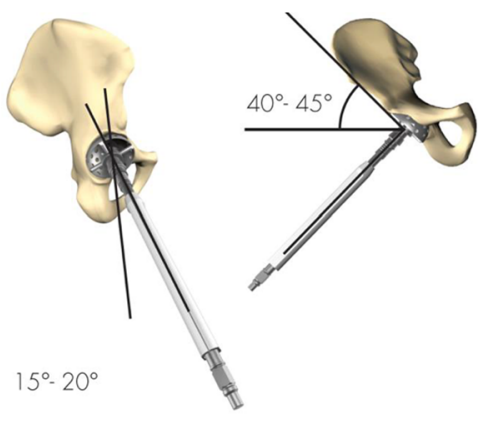

Fig. 2 Examples of images from the MyHip software: a Preoperative planning of the femoral component, $\mathbf{b}$ anteversion view, c preoperative planning of the acetabular component positioned with $45^{\circ}$ of inclination and $15^{\circ}$ of anteversion (Lewinnek safe zone)

the first one is focused on the affected side, the second one involves the contralateral side for comparison. This view is used to check the alignment between the two femurs, using the lesser trochanter as reference, Fig. 3.

A single, cementless implant system was used (Quadra-H System femoral components and Mpact System acetabular components, Medacta, Switzerland).

Surgical guides and anatomical models were produced by Selective Laser Sintering technology (SLS). PA 2200, a type of nylon that is a special moulding material developed by German EOS for their SLS rapid prototyping equipment, was used for 3D printing. (EOS, Munich, Germany). The components were sterilised either using gamma irradiation and delivered to our hospital or autoclaved at the hospital before intra-operative use.

\section{Intra-operative assessment and use of PSI}

For each patient, the planned sizes of acetabular and femoral components were recorded and compared to the actual sizes used at surgery. Complications such as intra-operative femoral fracture was also recorded.
During surgery, a patient-specific instrument guide was used to position the cup and cut the femoral neck, Fig. 4.

The acetabular guide was 3D printed to match the acetabular rim. Once seated into the acetabulum two pins were inserted through attached drill sleeves. The guide was then removed, leaving the two pins to act as either a constrained or unconstrained guide to reaming and component placement. The femoral PSI guide was 3D printed to fit the contours of the femoral head-neck junction (ie, no cartilage present at this junction). Once seated, it was secured with two threaded pins. The femoral neck cut was then completed using a standard method, with the saw blade flush on the cutting surface of the guide to deliver a femoral cut at the planned angle and location. The planned cut angle was $45^{\circ}$ from the piriformis fossa to the anatomical axis of the femur to match the etched mark on the femoral stem (also $45^{\circ}$ to the long axis of the stem). The femoral canal was prepared by the surgeon using the instructions for use provided by the implant manufacturer. The canal was opened using a starter reamer and femoral stem rasps, with sequentially increasing sizes, so

\section{a}

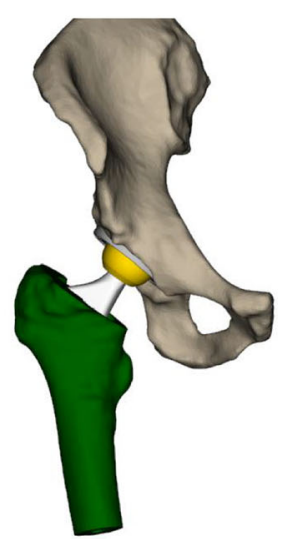

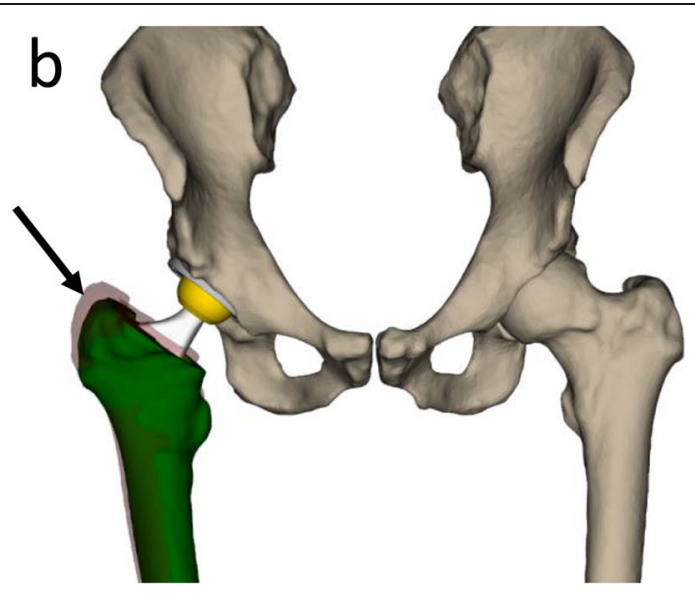

Fig. 3 Example of (a) the global view and (b) leg length view after planning. While the first displays the affected side, the second one involves the contralateral side too. This view is used to check the alignment between the two femurs, using the lesser trochanter as reference. The red model (arrow) represents the pre-op position and the green is the post-op one 

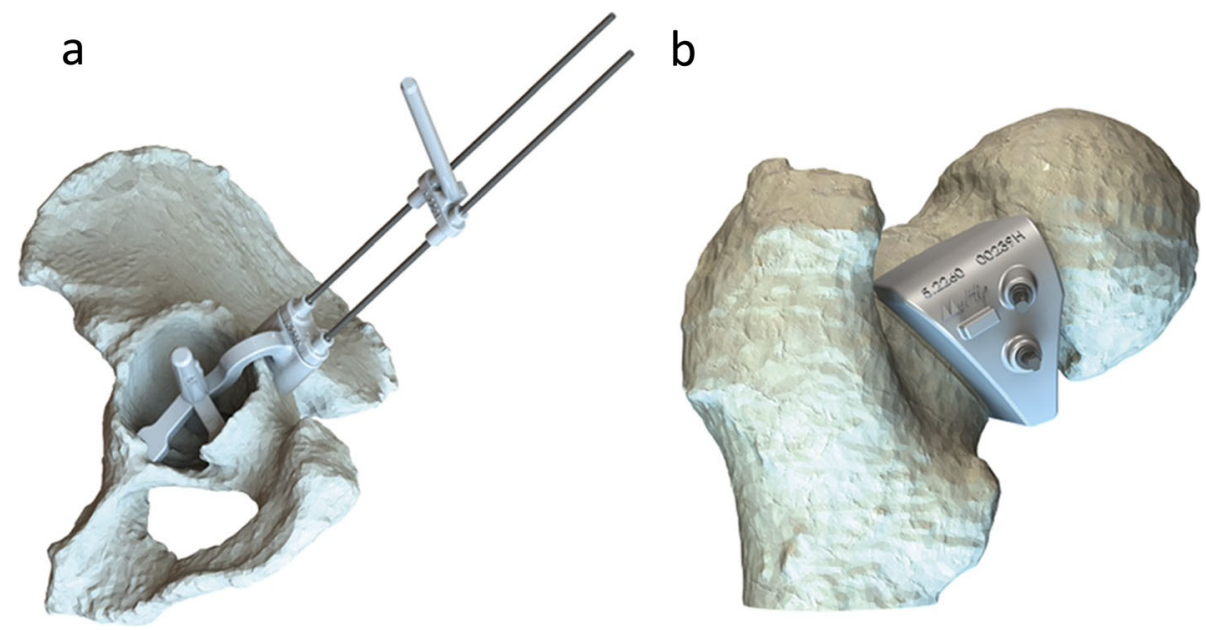

Fig. 4 The acetabular and femoral PSI guides. The acetabular guide is seated into the acetabulum, and two pins are inserted through attached drill sleeves. The guide is removed, leaving the two pins to act as either a constrained or unconstrained guide to reaming and component placement (a). The femoral guide has a contoured fit to the femoral neck/head and is kept in place for the neck cut by two intraosseous pins (b) [13]

that the etched stem marker was level with the cut surface of the femur and the rasp was secure when tested by twisting. The stem was then press-fitted and tested by twisting the implant within the femur and confirming that this did not cause movement between the stem and the bone.

\section{Post-operative radiological evaluation}

All patients underwent conventional standing anteroposterior (AP) radiographs of the pelvis and hip postoperatively. The surgeon assessed size and position of cup and stem as well as restoration of leg length. Number of dislocations and revisions were recorded.

\section{Statistical analysis}

Statistical analysis was performed using Prism 7 (GraphPad Software, San Diego, CA, USA). Paired t-test test was used to determine the difference between the two groups. The reproducibility of planned and implanted cup and stem sizes was determined using the Pearson's correlation coefficient. The level of significance for all statistical analyses was $p<0.05$.

\section{Results}

\section{Feasibility study}

Stem and cup components used were within 1 size of the planned. This enabled us to safely optimize the size of the inventory for the pilot study. The feasibility study created the rules for a safe reduction in implant inventory: 1) the planned size of stem, plus one size above and below, and duplicated for all neck options; 2) the planned size, plus one size above and below, of cup, cup liners (both polyethylene and polyethylene hooded), and femoral heads (all 4 neck lengths for both 32 and $36 \mathrm{~mm}$ because cups cross boundary between 32 and $36 \mathrm{~mm}$ ).

\section{Pilot study}

The pilot study showed: 1) 3D surgical planning correctly predicted sizes in $93 \%(42 / 45)$ of the femoral components and $89 \%(40 / 45)$ of the acetabular components; 2) a reduction in the implant inventory size from 101 to 39 components (a $61 \%$ reduction in inventory size); 3 ) good surgical outcomes with no difference between control and experimental groups and importantly, all patients in the group with a reduced inventory size had appropriately sized implants.

\section{Accuracy of 3D-CT planning}

All 45 patients had a cup or stem size within one size of planned. The achieved stem size corresponded to the preoperatively planned size in $93 \%(42 / 45)$ of cases. There was a high correlation between the planned and achieved stem sizes $(r=0.99, p<0.0001)$. CT planning correctly predicted size in $89 \%(40 / 45)$ of the acetabular cup components. The 5 discrepancies had cups that were undersized by one size when compared to the planned size. There was a high correlation between the planned and achieved cup sizes $(r=0.98, p<0.0001)$, Fig. 5 .

Accuracy of pre-operative CT planning increased over time, Fig. 6.

Head diameter was accurately determined in the majority of the cases (80 to $90 \%$ ), Fig. 7.

\section{Inventory assessment outcome}

No femoral fracture occurred during surgery and there were no errors attributed to a missing implant.

The full inventory contained 101 implants for the Quadra stem and Mpact Cup system. This was reduced to 39 implants following the feasibility study: a two third reduction (61\%), Fig. 8. 


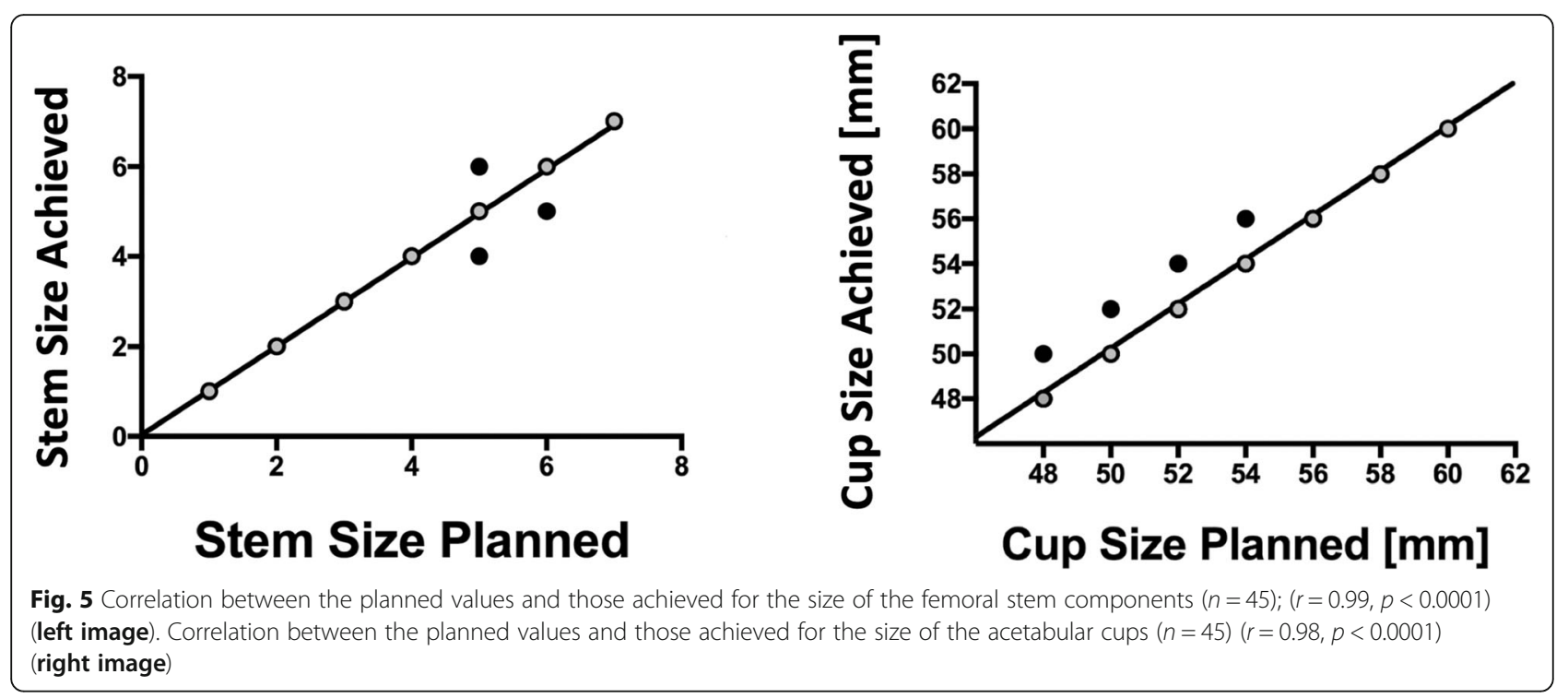

\section{Surgical outcome}

The post-operative course was uneventful for all patients $(n=45)$. Standard post-op 2D radiography showed satisfactory restoration of leg length and femoral offsets Fig. 9.

There was one revision operation. This was for cup loosening following a previous acetabular fracture in a patient with protrusio and was not related to the implant size. There were no other revision operations for any cause.

There were two dislocations, which occurred as a result of excessive range of motion: deep hip flexion at 5 weeks post operative; and a deep "child's pose" during yoga at 12 months post operative. Both of these cases

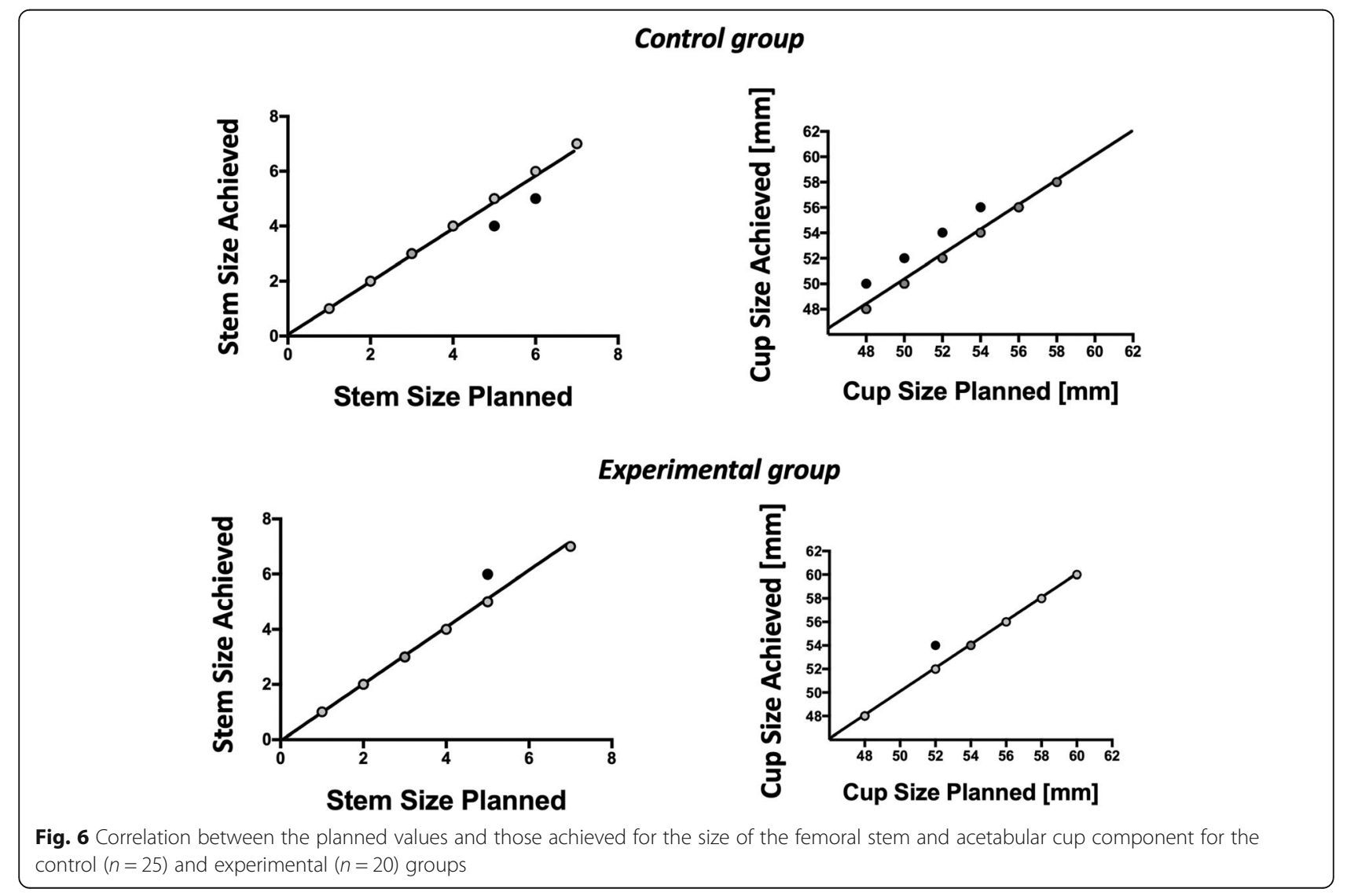




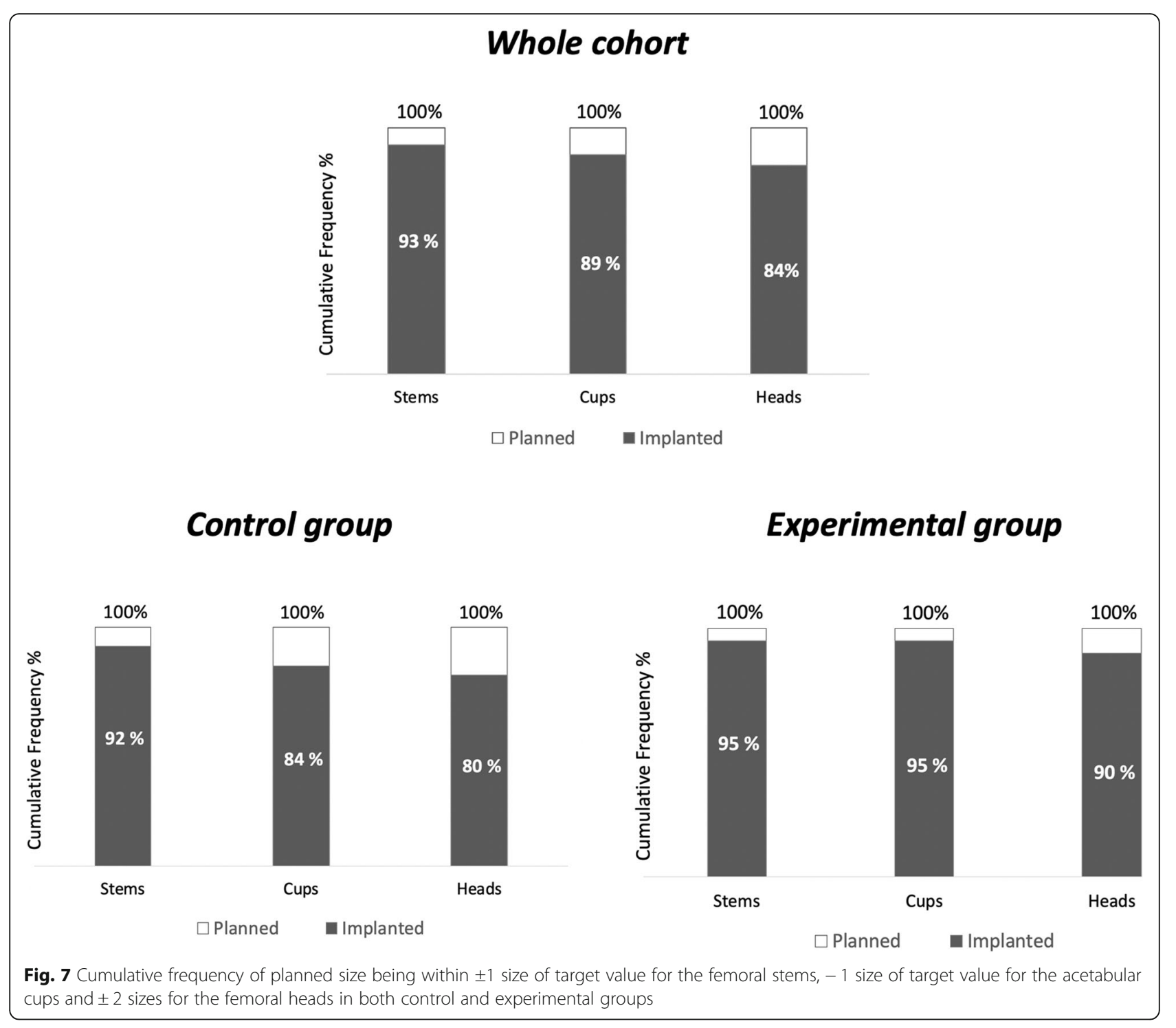

were treated with one closed reduction procedure for each, post reduction CT showed a satisfactory position, and clinical outcomes were good (both had an OHS of 48 out 48 with no further dislocations at last review, which was 12 months and 4 months post dislocation).

\section{Discussion}

THA is one of the most successful and cost effective medical advances of the last 50 years [22]; the implant is the most expensive supply item for joint replacement $[1$, 5]. The current increase in demand is driving the rationale for cost savings $[1,4]$.

The modern approach to THA involves a more targeted treatment relying on the use of advanced image modalities for both diagnosis and treatment. Preoperative 3D surgical planning helps determine the correct implant size [18, 23], component orientation and fixation, achieve restoration of femoral offset and limb length $[9,10]$. Moreover it enables the use of customised PSI which is sometimes an incorporated step in specialized planning software [24]. Accurate prediction of implant size during preoperative planning is an important factor for successful reconstruction in THA, to avoid intraoperative or postoperative complications. In addition, the accuracy of size prediction may facilitate inventory cost savings and reduce the risk of opening (and thus wasting) the packaging of an implant of the wrong size [25].

Aim of this study was to better understand the impact of the use of 3D surgical planning and PSI on hip implant inventory. This is the first study to investigate the use of CT-based planning software to optimise implant inventory in THA. We found that implant size prediction by $3 \mathrm{D}$ planning was accurate in up to $95 \%$ of the cases, which enabled us to safely reduce the 


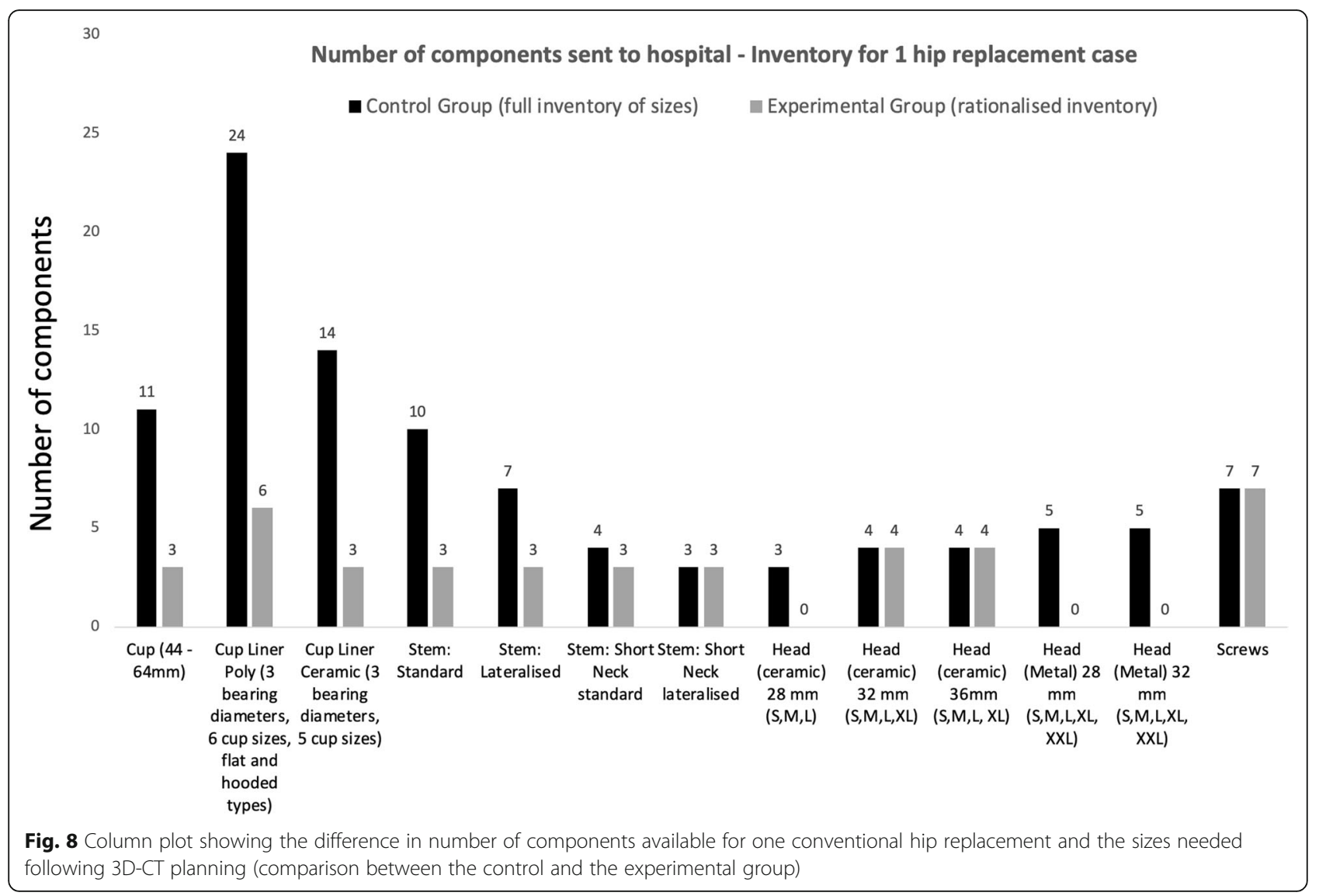

inventory size by up to $61 \%$. There were no complications relative to implant size.

3D planning correctly predicted implant size in $93 \%$ $(42 / 45)$ of the femoral components and 89\% (40/45) of the acetabular components. Stem and cup variability was within one-size in the feasibility study, which gave us the confidence to optimize the inventory for the pilot study.

The ability of 3D planning to predict implant size was up to 2.5 -fold superior to that reported when $2 \mathrm{D}$ templating is used (accurate in about a third of cases) $[8,26,27]$. A number of studies on cementless THAs have evaluated the accuracy of the preoperative planning by $2 \mathrm{D}$ or $3 \mathrm{D}$ templating. The agreement with cup size prediction was found to be between 20 and $42.2 \%$ with $2 \mathrm{D}$ planning and $86-96 \%$ with $3 \mathrm{D}$ planning, while the agreement with stem size prediction was 19$68.8 \%$ with $2 \mathrm{D}$ planning, $52-100 \%$ with $3 \mathrm{D}$ planning $[25,27]$.

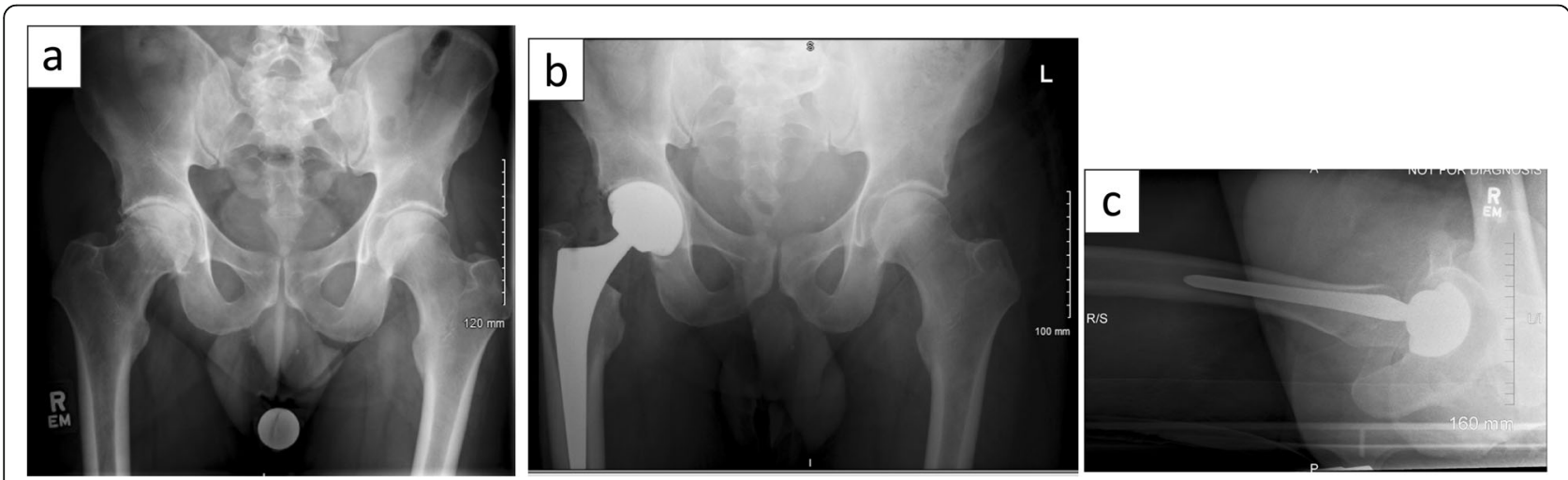

Fig. 9 a Pre-operative AP radiograph and $\mathbf{b}, \mathbf{c}$ post-operative radiographic AP and lateral views of the patient in standing and sitting position showing a satisfactory alignment of the prosthesis 
Our results are in accordance with the existing literature, Table 2.

2D templating is still the most commonly used planning method, despite its lower accuracy when compared to CT planning $[15,16]$, particularly when using cementless components [31,32] over cemented implants [9, 33] and the magnifications issues associated with conventional $2 \mathrm{D}$ radiographs.

A number of barriers hindrance the widespread use of new technology.

The use of CT data introduces new issues related to how best to display and use all information in order to provide surgeons with a user-friendly interface [34]. Another barrier to large adoption of CT planning is the learning curve associated with new technology and systems. Moreover, CTbased planning platforms that are not tied to an implant manufacturer, have recently been introduced to the European and US marketplace, but their uptake has been limited due to software and imaging costs, radiation dose and a lack of standardized optimized imaging protocols.

The use of 3D surgical planning has been largely limited due to the associated ionizing radiation exposure to the patient, limited availability of generic software planning solutions, high cost and limited availability of preoperative CT imaging protocols. Recent advances in lowdose CT technology tailored for the specific purpose of planning orthopaedic surgery have made justifying the radiation exposure to the patient considerably easier [21, 28]. The new imaging protocols include imaging of the hip, knee and ankle regions at a comparatively lower effective radiation dose without a significant trade off in image quality $[21,28]$. These protocols have seen a fourfold reduction in radiation and can be as low as equivalent to 3 pelvic radiographs [35], enhanced further by the introduction of orthopaedic metal artefact reduction (MARS) sequences on many modern CT scanners, allowing suppression of noise artefacts produced by preexisting metal implants.

Our results show a reduction in the size (up to 61\%) of implant inventory based on the use that is conventionally adopted when the cases are not $3 \mathrm{D}$ planned for our single surgeon series.

3D surgical planning aids surgical planning and implant sizing with greater accuracy, crucial for good functional hip reconstruction; it therefore can help minimizing the number of surgical trays used during the operation [15, 36, 37]. 3D planning enables a reduction in intraoperative guesswork, and allows for an optimised implant inventory with the potential to reduce the costs to the both manufacturer and hospital without additional risk [23]. However, it is currently unknown what effect CT planning has on the size of hip implant inventory in hospitals.

As the technological advancements in the field of orthopaedics have led to the introduction and development of sophisticated tools, 3D surgical planning is a promising field in which many more developments can be expected providing a quicker and more accurate surgery and the development of an optimal inventory of implants with benefits for all the parts involved in the chain.

We acknowledge limitations. First, we evaluated only one design of implant for both acetabular and femoral components, therefore our findings relative to the reduction in inventory size cannot be generalized to every $3 \mathrm{D}$ planning platform and implant type. They can be used as a baseline comparison for future studies aimed at quantifying inventory size reduction. Secondly, this study was based on a series of cases performed by one experienced consultant orthopaedic surgeon, therefore it is unknown whether similar results can be expected for surgeons with different level of experience and using different surgical approaches.

Table 2 Rate of prediction for cup and stem size when using 3D planning

\begin{tabular}{|c|c|c|c|c|c|c|c|}
\hline \multirow{2}{*}{$\begin{array}{l}\text { Reference } \\
\text { (Viceconti, Lattanzi et al. 2003) [15] }\end{array}$} & \multirow{2}{*}{$\begin{array}{l}\text { Subjects } \\
\text { n }\end{array}$} & \multirow{2}{*}{$\begin{array}{l}\text { Surgical } \\
\text { Indication } \\
\text { DDH (65.6\%) }\end{array}$} & \multirow{2}{*}{$\begin{array}{l}\text { Planning } \\
\text { Software } \\
\text { Hip-Op }\end{array}$} & \multirow{2}{*}{$\begin{array}{l}\text { Cemented/C.less } \\
\text { Total Hip System } \\
\text { C.less }\end{array}$} & \multirow{2}{*}{$\begin{array}{l}\text { Stem Modularity } \\
\text { (Yes/No) } \\
Y\end{array}$} & \multicolumn{2}{|c|}{$\begin{array}{l}\text { Size Prediction } \\
\text { (\%) } \\
\text { Stem-Cup }\end{array}$} \\
\hline & & & & & & 51.7 & 65.5 \\
\hline (Sariali, Mouttet et al. 2009) [17] & 223 & $\mathrm{OA}$ & HIP-PLAN & C.less & Y & 94 & 86 \\
\hline (Huppertz, Radmer et al. 2011) [28] & 92 & NA & 3D-Hip Plan ${ }^{\oplus}$ & 99\% C.less & Y & NA & NA \\
\hline (Sariali, Mauprivez et al. 2012) [16] & 60 & $\mathrm{OA}$ & Hip-Plan ${ }^{T M}$ & C.less & Y & 100 & 96 \\
\hline (Hassani, Cherix et al. 2014) [18] & 50 & NA & HIP-PLAN & C.less & Y & 100 & 94 \\
\hline (Inoue, Kabata et al. 2015) [23] & 57 & $\mathrm{DDH}$ & ZedHip & C.less & N & 65 & 92 \\
\hline (Mainard, Barbier et al. 2017) [29] & 31 & $\mathrm{OA}$ & hipEOS ${ }^{\mathrm{TM}}$ & C.less & N & $84^{\mathrm{a}}$ & $92^{\mathrm{a}}$ \\
\hline (Ogawa, Takao et al. 2018) [25] & 111 & $\mathrm{DDH}$ & CT-Based Hip Navigation System & C.less & N & 86 & 94 \\
\hline (Wako, Nakamura et al. 2018) [30] & 46 & OA (78\%) & ZedHip & C.less & Y & $92^{\mathrm{a}}$ & $90^{\mathrm{a}}$ \\
\hline Current Study & 45 & $\mathrm{OA}$ & MyHip & C.less & $N$ & 93 & 89 \\
\hline
\end{tabular}

$D D H$ Developmental Dysplasia of the Hip; NA Not Available; OA Osteoarthritis

${ }^{a}$ : within one size 


\section{Conclusion}

Planning of hip arthroplasty surgery on a $3 \mathrm{D}$ virtual CTbased model is useful to surgeons to help predict the size of the implants to be used in the operating room. The Medacta MyHip system used in this study can accurately predict component size for the femur and the acetabulum.

Based on the experience with our single surgeon series, there was a considerable reduction in the associated inventory use in the $\mathrm{CT}$ planned series vis-à-vis with the non-CT planned cases.

We envisage the potential for a phased introduction of a reduced hip implant inventory, and this should probably result in all sizes between 2 -sizes below planned up to 2-sizes above planned.

CT planning may become more widely adopted as both manufacturer and hospital seek to be more cost effective in the delivery of hip arthroplasty. A longer-term study is needed to help determine the degree of generalizability of the findings to the available planning software solutions, surgeons and implant manufacturers.

\section{Acknowledgements}

This research study was funded by the Medical Research Council (MRC), The Maurice Hatter Foundation, the RNOH Charity, the Rosetrees Trust, the Stoneygate Trust and by researchers at the National Institute for Health Research University College London Hospitals Biomedical Research Centre.

\section{Authors' contributions}

Study Design: $A H, J H, A D$. Data collection: $A H, J H, A D$. Data analysis: $A H, J H$, AD. Literature search: $A H, A D, H H$. Data interpretation: $A H, J H, A D, H H$. Manuscript writing: $A H, J H, A D, H H$. The author(s) read and approved the final manuscript.

\section{Funding}

This research did not receive any specific grant from funding agencies in the public, commercial, or not-for-profit sectors.

\section{Availability of data and materials}

The datasets used and/or analysed during the current study are available from the corresponding author on reasonable request.

\section{Ethics approval and consent to participate}

We confirm that all investigations were conducted in conformity with ethical principles of research and that institutional approval was obtained (SE16.020).

\section{Consent for publication}

'Not applicable'

\section{Competing interests}

The authors have no potential conflicts of interest to report.

Received: 17 April 2020 Accepted: 28 August 2020

Published online: 23 September 2020

\section{References}

1. Healy WL, lorio R. Implant selection and cost for total joint arthroplasty: conflict between surgeons and hospitals. Clin Orthop Related Res. 2007;457: 57-63.

2. Kurtz S, Mowat F, Ong K, Chan N, Lau E, Halpern M. Prevalence of primary and revision total hip and knee arthroplasty in the United States from 1990 through 2002. JBJS. 2005;87(7):1487-97.

3. Kurtz SM, Lau E, Ong K, Zhao K, Kelly M, Bozic KJ. Future young patient demand for primary and revision joint replacement: national projections from 2010 to 2030. Clin Orthop Related Res. 2009;467(10):2606-12.
4. Nichols Cl, Vose JG. Clinical outcomes and costs within 90 days of primary or revision total joint arthroplasty. J Arthroplasty. 2016:31(7):1400-6 e1403.

5. Barber TC, Healy WL. The hospital cost of total hip arthroplasty. A comparison between 1981 and 1990. JBJS. 1993;75(3):321-5.

6. Kumar PGA, Kirmani SJ, Humberg H, Kavarthapu V, Li P. Reproducibility and accuracy of templating uncemented THA with digital radiographic and digital TraumaCad templating software. Orthopedics. 2009;32:11.

7. Steinberg EL, Shasha N, Menahem A, Dekel S. Preoperative planning of total hip replacement using the TraumaCad ${ }^{\mathrm{TM}}$ system. Arch Orthop Trauma Surg. 2010;130(12):1429-32.

8. Carter LW, Stovall DO, Young TR. Determination of accuracy of preoperative templating of noncemented femoral prostheses. J Arthroplast. 1995;10(4): 507-13.

9. Eggli S, Pisan M, Müller M. The value of preoperative planning for total hip arthroplasty. J Bone Joint Surg Brit Vol. 1998;80(3):382-90.

10. Della Valle $A G$, Padgett DE, Salvati EA. Preoperative planning for primary total hip arthroplasty. JAAOS. 2005;13(7):455-62.

11. Chepelev L, Wake N, Ryan J, Althobaity W, Gupta A, Arribas E, Santiago L, Ballard DH, Wang KC, Weadock W. Radiological Society of North America (RSNA) 3D printing Special Interest Group (SIG): guidelines for medical 3D printing and appropriateness for clinical scenarios. 3D Printing Med. 2018; $4(1): 1-38$

12. Mitsouras D, Liacouras $P$, Imanzadeh A, Giannopoulos AA, Cai T, Kumamaru KK, George E, Wake N, Caterson EJ, Pomahac B. Medical 3D printing for the radiologist. Radiographics. 2015;35(7):1965-88.

13. Henckel J, Holme TJ, Radford W, Skinner JA, Hart AJ. 3D-printed patientspecific guides for hip Arthroplasty. JAAOS. 2018;26(16):e342-8.

14. Mitsouras D, Liacouras PC, Wake N, Rybicki FJ. RadioGraphics update: medical 3D printing for the radiologist. RadioGraphics. 2020;40(4):E21-3.

15. Viceconti M, Lattanzi $R$, Antonietti B, Paderni S, Olmi R, Sudanese A, Toni A. CT-based surgical planning software improves the accuracy of total hip replacement preoperative planning. Med Eng Phys. 2003:25(5):371-7.

16. Sariali E, Mauprivez R, Khiami F, Pascal-Mousselard H, Catonné Y. Accuracy of the preoperative planning for cementless total hip arthroplasty. A randomised comparison between three-dimensional computerised planning and conventional templating. Orthop Traumatol Surg Res. 2012:98(2):151-8.

17. Sariali E, Mouttet A, Pasquier G, Durante E, Catone Y. Accuracy of reconstruction of the hip using computerised three-dimensional pre-operative planning and a cementless modular neck. J Bone Joint Surg Brit Vol. 2009;91(3):333-40.

18. Hassani $H$, Cherix $S$, Ek ET, Rüdiger HA. Comparisons of preoperative threedimensional planning and surgical reconstruction in primary cementless total hip arthroplasty. J Arthroplast. 2014;29(6):1273-7.

19. Sim J, Lewis $M$. The size of a pilot study for a clinical trial should be calculated in relation to considerations of precision and efficiency. J Clin Epidemiol. 2012;65(3):301-8.

20. Lancaster GA, Dodd S, Williamson PR. Design and analysis of pilot studies: recommendations for good practice. J Eval Clin Pract. 2004;10(2):307-12.

21. Henckel J, Richards R, Lozhkin K, Harris S, y Baena FR, Barrett A, Cobb J: Very lowdose computed tomography for planning and outcome measurement in knee replacement: the imperial knee protocol. Bone Joint J 2006, 88(11):1513-1518.

22. Daigle ME, Weinstein AM, Katz JN, Losina E. The cost-effectiveness of total joint arthroplasty: a systematic review of published literature. Best Pract Res Clin Rheumatol. 2012:26(5):649-58.

23. Inoue D, Kabata T, Maeda T, Kajino Y, Fujita K, Hasegawa K, Yamamoto T, Tsuchiya $\mathrm{H}$. Value of computed tomography-based three-dimensional surgical preoperative planning software in total hip arthroplasty with developmental dysplasia of the hip. J Orthop Sci. 2015:20(2):340-6.

24. Schmid J, Chênes $C$, Chagué $S$, Hoffmeyer $P$, Christofilopoulos $P$, Bernardoni M, Charbonnier C. MyHip: supporting planning and surgical guidance for a better total hip arthroplasty. Int J Comput Assist Radiol Surg. 2015;10(10): 1547-56.

25. Ogawa T, Takao M, Sakai T, Sugano N. Factors related to disagreement in implant size between preoperative CT-based planning and the actual implants used intraoperatively for total hip arthroplasty. Int J Comput Assist Radiol Surg. 2018;13:1-12.

26. Shaarani SR, McHugh G, Collins DA. Accuracy of digital preoperative templating in 100 consecutive uncemented total hip arthroplasties: a single surgeon series. J Arthroplast. 2013:28(2):331-7.

27. Petretta R, Strelzow J, Ohly NE, Misur P, Masri BA. Acetate templating on digital images is more accurate than computer-based templating for total hip arthroplasty. Clin Orthop Related Res. 2015;473(12):3752-9. 
28. Huppertz A, Radmer S, Asbach P, Juran R, Schwenke C, Diederichs G, Hamm B, Sparmann M. Computed tomography for preoperative planning in minimal-invasive total hip arthroplasty: radiation exposure and cost analysis. Eur J Radiol. 2011;78(3):406-13.

29. Mainard D, Barbier O, Knafo Y, Belleville R, Mainard-Simard L, Gross J-B. Accuracy and reproducibility of preoperative three-dimensional planning for total hip arthroplasty using biplanar low-dose radiographs: a pilot study. Orthop Traumatol Surg Res. 2017;103(4):531-6.

30. Wako Y, Nakamura J, Miura M, Kawarai Y, Sugano M, Nawata K. Interobserver and intraobserver reliability of three-dimensional preoperative planning software in total hip arthroplasty. J Arthroplast. 2018;33(2):601-7.

31. Knight $J$, Atwater RD. Preoperative planning for total hip arthroplasty: quantitating its utility and precision. J Arthroplast. 1992;7:403-9.

32. Della Valle AG, Comba F, Taveras N, Salvati EA. The utility and precision of analogue and digital preoperative planning for total hip arthroplasty. Int Orthop. 2008;32(3):289-94.

33. Iorio R, Siegel J, Specht LM, Tilzey JF, Hartman A, Healy WL. A comparison of acetate vs digital templating for preoperative planning of total hip arthroplasty: is digital templating accurate and safe? J Arthroplast. 2009;24(2):175-9.

34. Lattanzi R, Viceconti M, Petrone M, Quadrani P, Zannoni C. Applications of $3 \mathrm{~d}$ medical imaging in orthopaedic surgery: Introducing the hip-op system. In: null: IEEE; 2002. p. 808

35. Boettner F, Sculco PK, Lipman J, Saboeiro G, Renner L, Faschingbauer M. The effect of a low radiation $C T$ protocol on accuracy of $C T$ guided implant migration measurement: a cadaver study. J Orthop Res. 2016;34(4):725-8.

36. Lattanzi R, Viceconti M, Zannoni C, Quadrani P. Toni A. Hip-Op: an innovative software to plan total hip replacement surgery. Med Inform Internet Med. 2002;27(2):71-83.

37. León-Muñoz VJ, Lisón-Almagro AJ, López-López M. Planning on CT-based $3 \mathrm{D}$ virtual models can accurately predict the component size for total knee arthroplasty. J Knee Surg. 2019.

\section{Publisher's Note}

Springer Nature remains neutral with regard to jurisdictional claims in published maps and institutional affiliations.

Ready to submit your research? Choose BMC and benefit from:

- fast, convenient online submission

- thorough peer review by experienced researchers in your field

- rapid publication on acceptance

- support for research data, including large and complex data types

- gold Open Access which fosters wider collaboration and increased citations

- maximum visibility for your research: over $100 \mathrm{M}$ website views per year

At $\mathrm{BMC}$, research is always in progress.

Learn more biomedcentral.com/submissions 